\title{
Fast Scene Text Detection with RT-LoG Operator and CNN
}

\author{
Dinh Cong Nguyen ${ }^{1,2}$, Mathieu Delalandre ${ }^{1}$, Donatello Conte ${ }^{1}$ and The Anh Pham ${ }^{2}$ \\ ${ }^{1}$ Tours University, Tours City, France \\ ${ }^{2}$ Hong Duc University, Thanh Hoa, Vietnam \\ \{mathieu.delalandre, donatello.conte $\} @ u n i v$-tours.fr, \\ \{nguyendinhcong, phamtheanh\}@hdu.edu.vn
}

\begin{abstract}
Keywords: $\quad$ Scene text detection, keypoint grouping, RT-LoG, character pattern.
Abstract: $\quad$ Text detection in scene images is of particular importance for the computer-based applications. The text detection methods must be robust against variabilities and deformations of text entities. In addition, to be embedded into mobile devices, the methods have to be time efficient. In this paper, the keypoint grouping method is proposed by first applying the real-time Laplacian of Gaussian operator (RT-LoG) to detect keypoints. These keypoints will be grouped to produce the character patterns. The patterns will be filtered out by using a CNN model before aggregating into words. Performance evaluation is discussed on the ICDAR2017 RRC-MLT and the Challenge 4 of ICDAR2015 datasets. The results are given in terms of detection accuracy and time processing against different end-to-end systems in the literature. Our system performs as one of the strongest detection accuracy while supporting at approximately 15.6 frames per second to the HD resolution on a regular $\mathrm{CPU}$ architecture. It is one of the best candidates to guarantee the trade-off between accuracy and speed in the literature.
\end{abstract}

\section{INTRODUCTION}

Scene text detection is a key topic in the literature (Long et al., 2018) since several years ago due to its influence in the real-life. Text detection can be applied in a wide range of applications such as traffic sign recognition, blind assistance, augmented reality and so on. However, detecting and localizing scene text still remains a challenge due to degradation. This covers different aspects such as the texture, illumination changes, the differences in languages, scales of characters and the background/foreground transitions Figure 1. Robust methods must be designed against variabilities, deformations of text entities.

Moreover, another crucial problem is to adapt the methods to be time-efficient such as they can embedded into mobile devices. This involves an almost complete reformulation of the methods to make them real-time in order to respect the time constraint for detection (Neumann and Matas, 2015).

The real-time methods in the literature apply a two-stage strategy for localization followed by text verification. The localization determines the positions of candidate text elements in the image at a low complexity level. The main goal is to process with a strong recall to not miss text elements. After that,

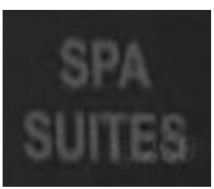

(a)

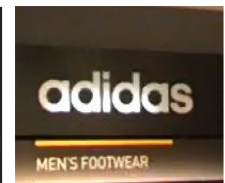

(b)

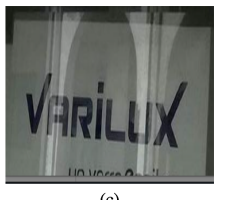

Figure 1: Examples of text in natural scenes with specific degradations (a) blurring (b) different sizes of character (c) illumination changes.

text verification specifies which candidate is text or not. It filters out the false alarms by using verification procedures. The two-stage strategy is opposite to the end-to-end strategy merging localization and verification (Long et al., 2018).

A core component of the two-stage strategy is the local operator. The local operator extracts candidate keypoints at the locations of text elements in the image. Different real-time operators have been proposed in the literature for scene text detection as the FASText operator (Busta et al., 2015), the Canny Text Detector (Cho et al., 2016) and the Maximally Stable Extremal Regions (MSER) operator (Gomez and Karatzas, 2014).

In this paper, we propose a new system applying a two-stage strategy for the real-time detection of scene text. Compared with the other systems in the litera- 
ture, our system applies in the first stage an RT-LoG operator. The RT-LoG operator is the real-time implementation of the Laplacian of Gaussian (LoG) operator (Fragoso et al., 2014; Nguyen et al., 2019). It is competitive for time processing and can be adapted to text detection. Furthermore, it provides meaningful scale-space and contrast information for detection.

This paper gives several key contributions.

- We propose a new grouping approach to embed the RT-LoG operator into a two-stage system.

- We highlight how the RT-LoG operator can support the full pipeline for scene text detection.

- We provide a performance evaluation where our overall system achieves one of the strongest detection accuracy of the literature, while requiring less than two orders of magnitude for the processing resources compared to our competitors.

The rest of paper is organized as follows. Section 2 presents our system. Then, the performance evaluation of the system is discussed in section 3. At last, section 4 will summarize and give out some perspectives. For convenience, Table 1 provides the meaning of the main symbols used in the paper.

\section{PROPOSAL APPROACH}

The general architecture is presented in Figure 2. At the first stage, we employ the RT-LoG operator to detect the keypoints among the strokes composing the characters. Next, a dedicated algorithm is proposed to group the keypoints using the spatial/scale-space representation of the RT-LoG operator. The grouping method results in Regions of Interest (RoIs) constituted of character patterns (characters, parts of characters, connected characters). These RoIs are verified into text/non text regions by a CNN. Before the veri-

Table 1: The main symbols used in the paper.

\begin{tabular}{c|c}
\hline Symbols & Meaning \\
\hline$x, y$ & Image coordinates \\
\hline$g=f(x, y)$ & $\begin{array}{c}f \text { the image function returning } \\
\text { the gray-level } g \text { at any location }(x, y)\end{array}$ \\
\hline$w$ & $\begin{array}{c}\text { Stroke width parameter } w \in\left[w_{\min }, w_{\max }\right] \\
\text { with }\left\{w_{\min }, \ldots, w_{\max }\right\} \text { the set of values }\end{array}$ \\
\hline$m$ & Scale parameter $m=\left(w_{\max }-w_{\min }\right)+1$ \\
\hline$h(x, y)$ & $\begin{array}{c}\text { Map of the responses } h \text { to all the pixels } \\
\text { in the image with } h \in[-1,1]\end{array}$ \\
\hline$\beta$ & A thresholding parameter \\
\hline$p=(x, y, g, h, w)$ & A keypoint \\
\hline$n, k$ & The number of keypoints with $k<<n$ \\
\hline$S$ & A set of keypoints $\left\{p_{1}, . ., p_{n}\right\}$ \\
\hline$Q \subseteq S$ & A subset of keypoints $\left\{p_{1}, . ., p_{k}\right\}$ \\
\hline$R$ & The number of Regions of Interest (RoIs) \\
\hline$R$ & A set of RoIs $R=\left\{R_{1}, ., R_{i}, . R_{q}\right\}$ \\
$R_{i}$ is a given RoI
\end{tabular}

fication, these RoIs are normalized using the RT-LoG and geometric features. This normalization relaxes the verification with the $\mathrm{CNN}$ from invariant problems such as the contrast, scale and orientation. A method for text line grouping is applied in the final stage. We will detail in following subsections.

\subsection{The RT-LoG Operator}

To start, we process the image with the RT-LoG operator to detect the keypoints constituting the characters Figure 3. Optimization of the operator is obtained with estimation of the LoG function and fast Gaussian convolution (Fragoso et al., 2014). Adaptation to stroke detection is given by the stroke model for scale-space representation (Nguyen et al., 2019; Liu et al., 2014).

The operator is controlled with a range of widths $w \in\left[w_{\min }, w_{\max }\right]$ of the strokes to detect. Within this range, $m=\left\{w_{\text {max }}-w_{\text {min }}\right\}+1$ is not only the number of stroke widths to detect but also the parameter of the scale-space problem. An additional parameter $\beta$ serves to threshold the operator responses and to tune the precision/recall of the detection.

The operator results in a set of $n$ keypoints $S=$ $\left(p_{1}, \ldots p_{i}, \ldots p_{n}\right)$ where a keypoint $p=(x, y, g, h, w)$ is given with $(x, y)$ the spatial coordinates, $g$ the gray level at the keypoint location, $h$ the operator response and $w$ the stroke width parameter. The operator response $h$ is either positive or negative depending the background/foreground transition of the character. Figure 3 gives an example of keypoint detection with the corresponding response map $h(x, y)$ providing the $h$ responses at every location $(x, y)$.

The RT-LoG operator provides meaningful scalespace and contrast information. This can drive a grouping method to obtain the character patterns and to guide a text verification stage. We will discuss these aspects in next subsections.

\subsection{The Spatial/scale-space Grouping}

The RT-LoG operator gives out, for each image, a set of keypoints belonging to the strokes composing the characters. These keypoints must be grouped together to constitute character patterns. The grouping of keypoints is well-known topic in the image processing and computer vision field (Dan et al., 2015). The main challenge here is to outline keypoints belonging to particular objects or RoIs in the image. Different strategies can be applied as the area-split with load imbalance, the clustering (e.g. K-means, Density-based spatial clustering), the grouping with 


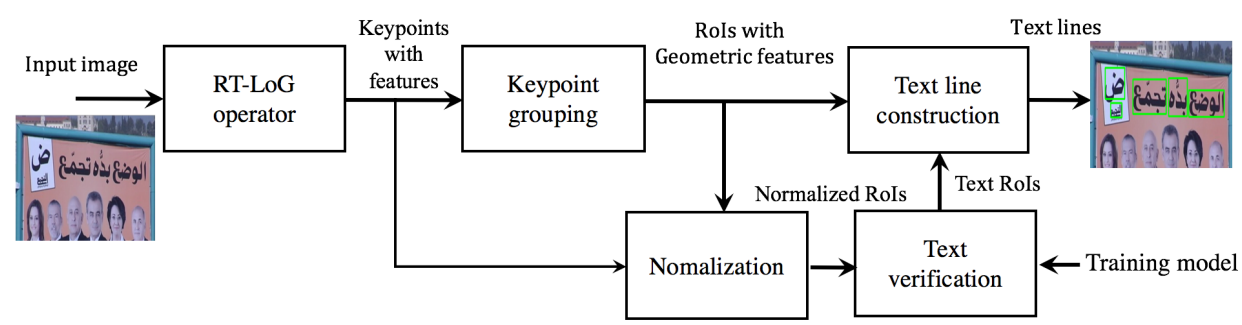

Figure 2: The detail of our approach.

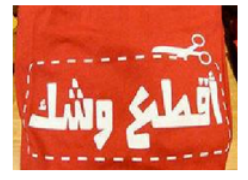

(a)

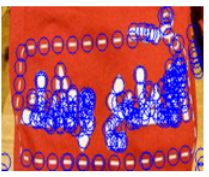

(b)

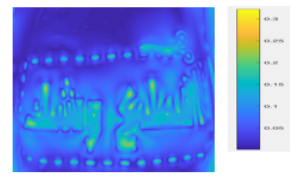

(c)
Figure 3: (a) input image (b) detected keypoints with circle/radius at $w / 2$ (c) the corresponding feature map $h(x, y)$.

machine learning (while characterizing the keypoints with descriptors) or the geometric consistency.

The grouping methods are dependent on the used features for the keypoints and the considered local operators. Several grouping methods for the LoG-based operators have been proposed in the literature Table 2. These methods target particular applications such as the general object detection or the scene text detection. The scale-space model is a key property to ensure a consistency while detecting the keypoints. Optimal model could be established for the stroke detection (Liu et al., 2014; Nguyen et al., 2019) but not for general object detection. In that case, the strategy of the grouping methods is driven by a high-level characterization of the keypoints with handcrafted features and/or machine learning methods.

At the best of our knowledge, a dedicated grouping method for the RT-LoG operator, while embedding the stroke model, has neither been investigated in the literature. In this paper, we propose a new approach to drive a consistent grouping with optimization using the spatial/scale-space representation of the RT-LoG operator. Our method uses three main steps as detailed in Figure 4. We will detail these steps in next paragraphs.

Foreground/background partitioning (step 1): in this step, we need to cope with both conditions in scene text detection such as a light character in a dark background producing minus responses and vice-versa. A keypoint $p=(x, y, g, h, w)$ is given with a normalized response of the operator $h$. This response $h \in[-1,1]$ results in two conditions of white $h \in[-1,0[$ and dark $h \in] 0,1]$ foregrounds, respectively. The keypoints belonging to a same character pattern are supposed to have a similar response. This
Table 2: The overview of the grouping methods for LoGbased operators.

\begin{tabular}{|c|c|c|}
\hline References & Methods & Applications \\
\hline (Dong et al., 2015) & $\begin{array}{l}\text { The stroke width values are } \\
\text { locally calculated and gathered } \\
\text { into SWT map. }\end{array}$ & $\begin{array}{c}\text { Text } \\
\text { detection }\end{array}$ \\
\hline (Mao et al., 2013) & $\begin{array}{l}\text { The geometric context of } \\
\text { SIFT keypoints is discovered. }\end{array}$ & $\begin{array}{c}\text { Text } \\
\text { detection }\end{array}$ \\
\hline (Shivakumara and Phan, 2010) & $\begin{array}{l}\text { K-means clustering is used } \\
\text { to identify candidate text regions } \\
\text { based on the maximum difference. }\end{array}$ & $\begin{array}{c}\text { Text } \\
\text { detection }\end{array}$ \\
\hline (Xu et al., 2016) & $\begin{array}{c}\text { Keypoints are clustered into } \\
\text { different group by mean-shift } \\
\text { algorithm and geometrical closeness. }\end{array}$ & $\begin{array}{c}\text { Object } \\
\text { detection }\end{array}$ \\
\hline (Fernndez-Carams et al., 2014) & $\begin{array}{l}\text { Vertical lines is produced by the } \\
\text { Haar-like features and Integral image. }\end{array}$ & $\begin{array}{c}\text { Object } \\
\text { detection }\end{array}$ \\
\hline
\end{tabular}

step splits the set of keypoints into two subsets based on their responses.

Scale-space partitioning and grouping (steps 2,3): the RT-LoG operator is scale-invariant and provides a stroke width parameters $w$ for each of the detected keypoint. This is an useful information that can be used as a local threshold to group the keypoints. The RT-LoG operator applies a Non-maximum suppression (NMS) within $3 \times 3$ local neighborhood. This guarantees an overlapping between the keypoints, while setting the operator with $w_{\min } \geq 2$.

The grouping performs for each of the keypoint $p_{i}$ in the subsets obtained from the step 1. A keypoint $p_{i}$ is grouped with a keypoint $p_{j}$, with $i, j \in[1, n]$, if their Euclidean distance fits with Eq (1), illustrated in Figure 5. In that case, the label of the keypoint $p_{i}$ is propagated to the keypoint $p_{j}$.

$$
\left\|p_{i}-p_{j}\right\|_{2} \leq \frac{w_{i}+w_{j}}{2}
$$

Similar to (Cabaret and Lacassagne, 2017), the overall grouping must applies forward/backward requests and propagation to merge the labels between the keypoints. The requests could be time-consuming depending significantly on the used request algorithm and the number of keypoints. For optimization, the keypoints can indexed with a KD-tree based DBSCAN (Vijayalaksmi and Punithavalli, 2012). The method is known as one of the fastest in the literature for the regular range searching problem. The overall grouping can be achieved with $n \log (n)$ comparisons. 
We propose here a strategy for optimization taking into account the specificity of the detection problem with the RT-LoG operator. A large number of keypoints belonging to a character pattern has a close stroke width Figure 6 (a). We use this property to group at a low complexity level the keypoints within the same scale. After that, the grouping is extended to the in-between scales. We use here a two-stage strategy for grouping, as detailed below.

- Step 2: the keypoints are partitioned first into subsets according to their stroke width parameter $w \in\left[w_{\text {min }}, \ldots, w_{\text {max }}\right]$. This is obtained with a linear scan of the overall set $S=\left(p_{i}, \ldots, p_{n}\right)$ at a complexity $O(n)$. A subset is noted as $Q=\left(p_{1}, . ., p_{k}\right)$ where $k<<n$. Every subset $Q$ is indexed with KD-tree based DBSCAN to get cluster components corresponding to the character pattern Figure 6 (b). This process has a complexity $k \log (k)$ for every subset $Q$. Each cluster is assigned with an ID.

- Step 3: the grouping is extended to the in-between scales Figure 6 (c). For optimization, clusters from the different scales will be compared. The comparison is stopped at the first matching among clusters. The forward/backward propagation is applied to merge the labels and the clusters to get the set of RoIs $R=\left\{R_{1}, ., R_{i}, ., R_{q}\right\}$.

Finally, we compute a set of geometric features from the keypoints belonging to a RoI to get the centroid, the area/perimeter, the bounding box and the orientation. These features will be used in the scene text detection pipeline to drive the image normalization and text line construction Figure 2.

\subsection{Verification and Text Line Construction}

The grouping algorithm results in the detection of RoIs. As the RT-LoG operator detects strokes, the detected RoIs could be either character patterns or background elements from the natural scene image. The RoIs must be classified to filter out the character patterns from background. This is the text verification problem that takes part in the scene text detection.

The traditional approach for scene text detection is to apply hand-crafted features with classification. Re-

Table 3: CNN models for text verification.

\begin{tabular}{c|c|c|c}
\hline Image size & \multicolumn{3}{|c}{$(24 \times 24)-(32 \times 32)$} \\
\hline Layer No & Filter size & Filter No & Pooling \\
\hline Conv1 & $(5 \times 5)-(8 \times 8)$ & x20 - x96 & $(2 \times 2)-(5 \times 5)$ \\
Conv2 & $(2 \times 2)-(5 \times 5)$ & x50 - x256 & $(2 \times 2)$ \\
\hline
\end{tabular}

cently, $\mathrm{CNN}$ becomes prominent into the field where a main issue is to design end-to-end system (Long et al., 2018). When applying to text verification, the CNN classifies the RoIs of images to verify if a RoI belongs to a text part or not.

Several CNN models have been proposed in the literature for the text verification (Yang et al., 2015; Ray et al., 2016; Wang et al., 2018; Zhang et al., 2015; Ghosh et al., 2019). Table 3 gives a summary. The fundamental model is to process with two convolutional layers while using a ReLU function for the non-linearity and a max pooling for optimization. A fully-connected layer (FC) is used in the final stage for classification set with a softmax function. Some works have investigated deeper models using up to four convolutional layers as (Zhang et al., 2015).

For the sake of performance evaluation, we customize our model inspired from (Wang et al., 2018; Ghosh et al., 2019). It is produced with two convolutional layers and one FC layer. For each convolutional layer, ReLu activation and average pooling layer are followed.

A core problem for text verification is the variability character patterns. These patterns are not normalized and suffered from distortions Figure 7 (a) (d). Another problem comes from the look-like cursive characters that results in word patterns after detection and grouping Figure 7 (e). The normalization of these patterns to $32 \times 32$ images introduces other distortions as the down-scaling and the modification of the aspect ratio. This tends to burden the learning period of the CNN training for text verification.

To solve these problems, we apply an image normalization before to classify the character patterns with the CNN Figure 2. We use the RT-LoG and geometric features to drive the normalization as they provide meaningful information about the character patterns. Our process is detailed here.

- Skew correction: we use the geometric features to correct the skew of characters with image rotation Figure 7 (a).

- Background/foreground normalization: the keypoints belonging to a character pattern provide a common operator response $h$. This response is whether positive or negative depending on the background/foreground transition. We look for all the character patterns having a positive response $h>0$ and invert the image for normalization Figure 7 (b).

- Contrast normalization: the character patterns appear with different background/foreground amplitudes. This is a problem of contrast normalization that can be solved from the operator response $h$. Indeed, the operator response $h \in[-1,1]$, where 


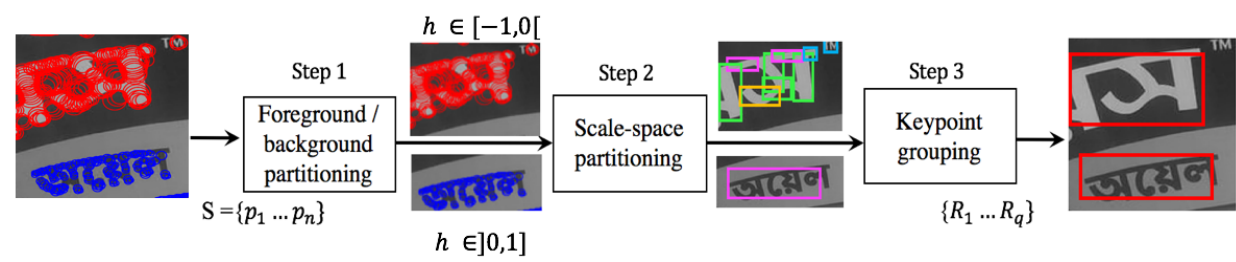

Figure 4: The detail of spatial/scale-space grouping method.

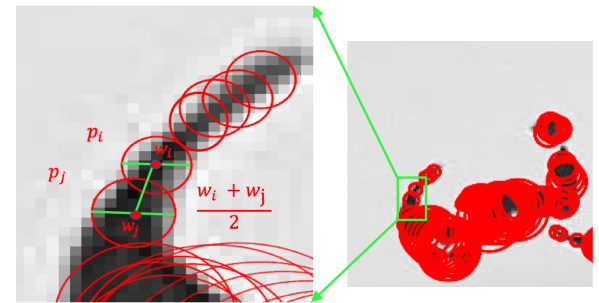

Figure 5: The grouping rule of two keypoints.

$|h|=1$ is obtained from bilevel black and white image having a maximum amplitude. We use the response $h$ and the $g$ values to get a lookup table to normalize the contrast Figure 7 (c).

- Scale normalization: the normalization of character patterns to $32 \times 32$ image distorts the aspect ratio Figure 7 (d). We use the average stroke width $\bar{w}$ of character patterns as a scale estimator. This estimator serves to compute a scale ratio $\overline{w_{0}} / \bar{w}$ with $\overline{w_{0}}$ an offline parameter obtained from the training database. The parameter $\overline{w_{0}}$ is fixed in order to embed the character patterns into a $32 \times 32$ image, as an average. If not, we split the character patterns into $32 \times 32$ patches while respecting an overlapping threshold Figure 7 (e).

In the final step, after verification with normalization, the close text regions must be grouped to get the text lines. Similar to (Cho et al., 2016), we use the method of minimum-area encasing rectangle to provide consistent bounding boxes.

\section{PERFORMANCE EVALUATION}

In this section, we present the performance evaluation of our system. Section 3.1 introduces the used datasets. Section 3.2 details the characterization metrics. Results are discussed in terms of detection accuracy and time processing in sections 3.3 and 3.4.

\subsection{Datasets}

Several public datasets have been proposed over the years for performance evaluation of text detec- tion methods. The ICDAR2017 and ICDAR2019 RRC-MLT are the two up-to-date datasets in literature (Nayef et al., 2017; Nayef et al., 2019). The ICDAR2019 RRC-MLT is a recent dataset and a slight update of the ICDAR2017 RRC-MLT ${ }^{1}$. For our performance evaluation, we have selected the ICDAR2017 RRC-MLT dataset where more comparative results are available in the literature. It includes 7200 training images, 1800 validation images and 9000 test images. The images are given at different resolutions (VGA, HD, Full-HD, Quad-HD, 4K). This dataset has a particular focus on the multi-lingual scene text detection in 9 languages and offers a deep challenge for scalability. Figure 8 shows some visual examples of images.

For the processing time, it is more common in the literature to use the Challenge 4 of ICDAR2015 dataset (Karatzas and Gomez-Bigorda, 2015). This dataset contains 1000 training images and 500 test images at HD resolution $(1280 \times 720)$.

\subsection{Characterization Metrics}

For the characterization metrics, we have followed the recommendations of the international contest (Nayef et al., 2017). The characterization is achieved at two levels while applying the Intersection over Union (IoU) criterion and computing the F-measure. The output of the text detection system is provided with bounding boxes. A detection (i.e. a true positive) is obtained if a detected bounding box has more than $50 \%$ overlap (the IoU criterion) with a bounding box in the groundtruth. The unmatched boxes in the detection and the groundtruth are false positives and negatives, respectively. The detection cases serve to compute the regular metrics precision $(\mathrm{P})$, recall $(\mathrm{R}), \mathrm{F}$ measure $^{2}(\mathrm{~F})$. Let's note that some degraded texts in the dataset are marked as "don't care" boxes and ignored in the evaluation process.

\footnotetext{
${ }^{1}$ The ICDAR2019 RRC-MLT dataset includes 1K images more on the existing set of $9 \mathrm{~K}$ images.

${ }^{2} \mathrm{We}$ are not detailing these aspects here and report to (Nayef et al., 2017).
} 


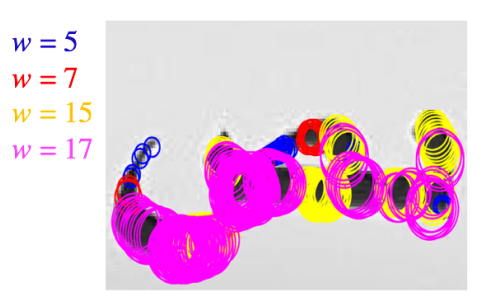

(a)

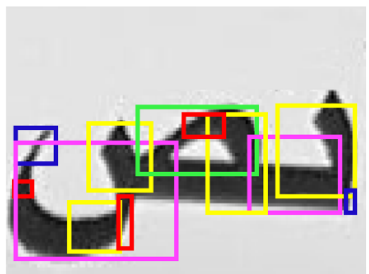

(b)

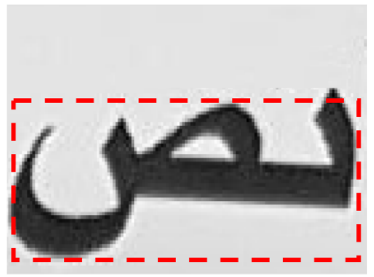

(c)

Figure 6: (a) Keypoints within the scale-space partitions (b) grouping within the scale-space partitions (c) grouping within in-between scale-spaces.

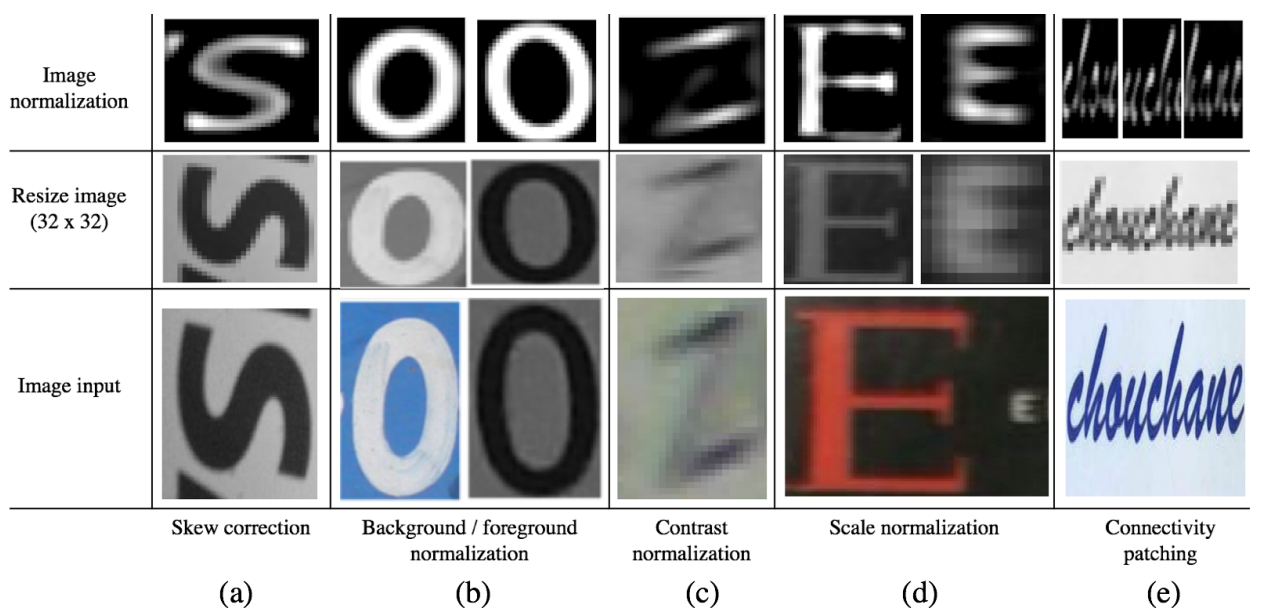

Figure 7: Image normalization.

Table 4: Comparison of methods

(P) Precision (R) Recall and (F) F-Measure on ICDAR2017 RRC-MLT.

\begin{tabular}{c|c|c|c|c}
\hline Rank & Methods & $\mathrm{P}(\%)$ & $\mathrm{R}(\%)$ & $\mathrm{F}(\%)$ \\
\hline 1 & PMTD (Liu et al., 2019) & $\mathbf{8 5 . 1 5}$ & 72.77 & $\mathbf{7 8 . 4 8}$ \\
\hline 2 & FCN-MOML (He et al., 2018) & 82.66 & 72.53 & 77.26 \\
\hline 3 & R-CNN-PAN (Huang et al., 2019) & 80 & 69.8 & 74.3 \\
\hline 4 & LOMO MS (Zhang et al., 2019) & 80.2 & 67.2 & 73.1 \\
\hline 5 & Proposed method with normalization & 65.2 & 82.1 & 72.6 \\
\hline 6 & MOSTD (Lyu et al., 2018b) & 74.3 & 70.6 & 72.4 \\
\hline 7 & Proposed method without normalization & 62.5 & $\mathbf{8 2 . 7}$ & 71.2 \\
\hline 8 & Fots (Liu et al., 2018) & 81.86 & 62.30 & 70.75 \\
\hline 9 & AF-RPN (Zhong et al., 2018) & 75 & 66 & 70 \\
\hline 10 & Attention Model (Wang et al., 2019) & 72 & 63.5 & 67.48 \\
\hline 11 & SCUT DLVClab1 (Nayef et al., 2017) & 80.3 & 54.5 & 65 \\
\hline
\end{tabular}



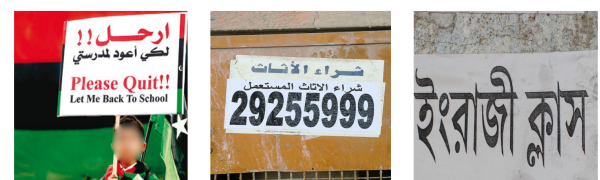

Figure 8: Images from the ICDAR2017 RRC-MLT dataset (Nayef et al., 2017).
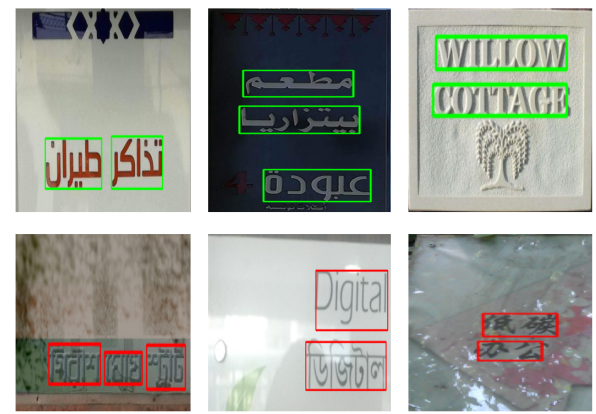

Figure 9: Text detection samples with (green) the true detections and (red) the missed-cases.

\subsection{Scene Text Detection}

Table 4 shows our evaluation in comparison with the state-of-the-art. For clarification, we have stood on the top 10 of competitive systems of the literature.

In addition, our system is given in two implementation with/without image normalization. Our goal here is to illustrate how the RT-LoG operator can support the CNN for classification.

As highlighted in Table 4, our system appears in the top 5 for the F-measure score. Furthermore, our system achieves the strongest recall score of the literature. This is ensured by the use of the RT-LoG operator and the dedicated grouping method, which allows a near complete detection of text elements. The normalization obtained with the RT-LoG operator results in a slight gap for the F-measure score from 1\%-2\%.

Visual examples of true and missed detections are shown in Figure 9. The operator fails to detect text in images with very low contrast. This can be explained by the real-time implementation of the LoG operator, that is not contrast invariant (Nguyen et al., 2019).

\subsection{Processing Time}

Table 5: Average processing time in milliseconds (ms) / amounts of pixels, keypoints and RoIs of each step of the proposed method.

\begin{tabular}{c|c|c}
\hline Types & HD & Data workflow \\
\hline Methods & $320 \mathrm{~ms}$ & 1.2 Mpixel \\
\hline RT-LoG & $662 \mathrm{~ms}$ & 5.2 Kkeypoints \\
\hline Opte-force KD-tree/DBSCAN & $258 \mathrm{~ms}$ & 5.2 Kkeypoints \\
\hline Verification & $336 \mathrm{~ms}$ & 90 RoIs \\
\hline
\end{tabular}

We characterize and compare in this section the processing time of our system against other systems in the literature. As discussed in section 3.1, the ICDAR2015 dataset has been used for testing as it is common for processing time evaluation.

We have computed first the processing time of each step of our method using a single thread/core implementation Table 5. Our implementation is done with the $\mathrm{C}++$ language and tested on the Mas-OS and an Intel(R) Core(TM) i7- 4770HQ CPU, $2.2 \mathrm{GHz}$ having a near 32 GFLOPS $\mathrm{SP}^{3}$ performance.

We provide in addition in Table 5 information about the data workflow in the pipeline ( the amounts of pixels, keypoints and RoIs). The optimization achieved by our grouping method results in an approximate two to three times for acceleration factor.

Compared to systems-based R-CNN for scene text detection (Liu et al., 2019), our system produces less RoIs. These RoIs are resized into $32 \times 32$ before verification. This explains the low-level processing time required by our text verification stage.

As highlighted the RT-LoG operator results in a large reduction of the data to process. Despite the different of amount of data, the processing time is close between the RT-LoG operator and the rest of pipeline (grouping with $\mathrm{CNN}$ ). This can be explained by the real-time implementation of the operator that ensures a strong optimization of the spatial convolution.

We have evaluated, in a second step, the Frame rate per second (FPS) of our system on the ICDAR2015 dataset Table 6. This evaluation has been done with a full parallelism support on the CPU while applying multi-core/threading. We provide in addition the FPS of the top systems in literature. For a fair comparison, Table 6 addresses the test architectures of different systems (either GPU or CPU) with their relative performances, as obtained from CPU/GPU benchmarks ${ }^{4}$.

Table 6: Frame rate per second (FPS) among methods on the Challenge 4 of ICDAR2015 dataset.

\begin{tabular}{c|c|c|c}
\hline $\begin{array}{c}\text { Processing } \\
\text { types }\end{array}$ & FPS & Architecture & $\begin{array}{c}\text { Performances } \\
\text { TFLOPS }\end{array}$ \\
\hline FOTs-RT(Liu et al., 2018) & 22.6 & $\begin{array}{c}\text { TITAN-Xp } \\
\text { GPU }\end{array}$ & 12.15 \\
\hline Ours & 15.6 & $\begin{array}{c}\text { CPU } \\
2.2 \text { GHz }\end{array}$ & $\mathbf{0 . 0 3 2}$ \\
\hline SSTD (He et al., 2017) & 7.7 & $\begin{array}{c}\text { TITAN X } \\
\text { GPUs }\end{array}$ & 6.691 \\
\hline EAST (Zhou et al., 2017) & 6.52 & $\begin{array}{c}\text { TITAN-Xp } \\
\text { GPU }\end{array}$ & 12.15 \\
\hline MTS(Lyu et al., 2018a) & 4.8 & $\begin{array}{c}\text { Titan Xp } \\
\text { GPU }\end{array}$ & 12.15 \\
\hline MOSTD(Lyu et al., 2018b) & 3.6 & $\begin{array}{c}\text { Tesla } \\
\text { K40m GPU }\end{array}$ & 5.046 \\
\hline
\end{tabular}

\footnotetext{
${ }^{3}$ Single Precision.

${ }^{4}$ www.techpowerup.com; www.cpubenchmark.net.
} 
As emphasized in Table 6, our system has the second highest FPS while processing with a difference of two orders of magnitude in term of processing resources. All the top systems in the literature perform with end-to-end CNN model requiring a GPU architecture. For clarification, Figure 10 presents a general comparison of the performance considering the F-measure scores, FPS and test architecture.

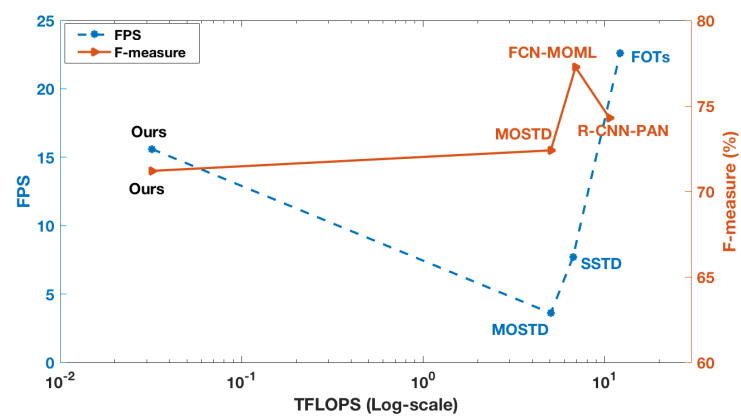

Figure 10: The performance considering the F-measure scores and FPS from scene text detection systems correspond to their architecture.

\section{CONCLUSIONS AND PERSPECTIVES}

This paper presents a new two-stage system for scene text detection. It applies in the first stage the RT-LoG operator. This operator supports the full pipeline for scene text detection. A dedicated algorithm is applied to group the keypoints into RoIs using the RT-LoG features. The RoIs are then classified into text/non text regions by a CNN. Before the verification, the RoIs are normalized with the RT-LoG features. This normalization relaxes the verification from invariant problems. The proposed system is in the top 5 for the F-measure score and achieves the strongest recall of the literature. It performs as one of the highest FPS rate while processing with a difference of two orders of magnitude in term of processing resources.

As a main perspective, the precision rate of the system can be consolidated. This can be obtained by making the RT-LoG operator contrast-invariant, to deal with the missed detection cases. Deeper CNN models could be applied to make more robust at the text verification stage. At last, acceleration of the RTLoG operator could be obtained by taking advantage of the Gaussian kernel distribution and decomposition with box filtering.

\section{REFERENCES}

Busta, M., Neumann, L., and Matas, J. (2015). Fastext: Efficient unconstrained scene text detector. In International Conference on Computer Vision (ICCV), pages 1206-1214.

Cabaret, L. and Lacassagne, L. (2017). Distanceless label propagation: an efficient direct connected component labeling algorithm for gpus. In International Conference on Image Processing Theory, Tools and Applications (IPTA), pages 1-6.

Cho, H., Sung, M., and Jun, B. (2016). Canny text detector: Fast and robust scene text localization algorithm. In International Conference on Computer Vision and Pattern Recognition (CVPR), pages 3566-3573.

Dan, G., Khan, M., and Fodor, V. (2015). Characterization of surf and brisk interest point distribution for distributed feature extraction in visual sensor networks. volume 17, pages 591-602.

Dong, W., Lian, Z., Tang, Y., and Xiao, J. (2015). Text detection in natural images using localized stroke width transform. In International Conference on Multimedia Modeling (ICMM), pages 49-58.

Fernndez-Carams, C., Moreno, V., and Curto, B. (2014). A real-time door detection system for domestic robotic navigation. Journal of Intelligent Robotic Systems, 76(1):119-136.

Fragoso, V., Srivastava, G., Nagar, A., and Li, Z. (2014). Cascade of box (cabox) filters for optimal scale space approximation. In International Conference on Computer Vision and Pattern Recognition Workshops (CVPRW), pages 126-131.

Ghosh, M., Mukherjee, H., Obaidullah, S., Santosh, K., Das, N., and Roy, K. (2019). Identifying the presence of graphical texts in scene images using cnn. In International Conference on Document Analysis and Recognition Workshops (ICDARW), volume 1, pages 86-91.

Gomez, L. and Karatzas, D. (2014). Mser-based real-time text detection and tracking. In International Conference on Pattern Recognition (ICPR), pages 3110 3115.

He, P., Huang, W., He, T., and Zhu, Q. (2017). Single shot text detector with regional attention. In International Conference on Computer Vision (ICCV), pages 3047 3055.

He, W., Zhang, X., Yin, F., and Liu, C. (2018). Multioriented and multi-lingual scene text detection with direct regression. Transactions on Image Processing (TIP), 27(11):5406-5419.

Huang, Z., Zhong, Z., Sun, L., and Huo, Q. (2019). Mask r-cnn with pyramid attention network for scene text detection. In Conference on Applications of Computer Vision (WACV), pages 764-772.

Karatzas, D. and Gomez-Bigorda, L. (2015). Icdar 2015 competition on robust reading. In International Conference on Document Analysis and Recognition (IC$D A R)$, pages 1156-1160.

Liu, J., Liu, X., Sheng, J., and Liang, D. (2019). Pyramid mask text detector. arXiv preprint:1903.11800. 
Liu, X., Liang, D., S.Yan, and Chen, D. (2018). Fots: Fast oriented text spotting with a unified network. In Conference on computer vision and pattern recognition (CVPR), pages 5676-5685.

Liu, Y., Zhang, D., Zhang, Y., and Lin, S. (2014). Real-time scene text detection based on stroke model. In International Conference on Pattern Recognition (CVPR, pages 3116-3120.

Long, S., He, X., and Ya, C. (2018). Scene text detection and recognition: The deep learning era. arXiv:1811.04256.

Lyu, P., Liao, M., .Yao, C., and Wu, W. (2018a). Mask textspotter: An end-to-end trainable neural network for spotting text with arbitrary shapes. In The European Conference on Computer Vision (ECCV).

Lyu, P., Yao, C., and W. Wu, S. Y. (2018b). Multi-oriented scene text detection via corner localization and region segmentation. In Conference on Computer Vision and Pattern Recognition (CVPR), pages 7553-7563.

Mao, J., Li, H., Zhou, W., Yan, S., and Tian, Q. (2013). Scale based region growing for scene text detection. In International conference on Multimedia (ACM), pages 1007-1016.

Nayef, N., Patel, Y., Busta, M., and .Chowdhury, P. (2019). Icdar2019 robust reading challenge on multi-lingual scene text detection and recognition-rrc-mlt-2019. arXiv preprint: 1907.00945.

Nayef, N., Yin, F., Bizid, I., and Choi, H. (2017). Icdar2017 robust reading challenge on multi-lingual scene text detection and script identification-rrc-mlt. In International Conference on Document Analysis and Recognition (ICDAR), volume 1, pages 1454-1459.

Neumann, L. and Matas, J. (2015). Real-time lexicon-free scene text localization and recognition. Transactions on pattern analysis and machine intelligence (PAMI), 38(9):1872-1885.

Nguyen, D., Delalandre, M., Conte, D., and Pham, T. (2019). Performance evaluation of real-time and scale-invariant log operators for text detection. In International Conference on Computer Vision, Imaging and Computer Graphics Theory and Applications (VISAPP), pages 344-353.

Ray, A., Shah, A., and Chaudhury, S. (2016). Recognition based text localization from natural scene images. In International Conference on Pattern Recognition (ICPR), pages 1177-1182.

Shivakumara, P. and Phan, T. (2010). A laplacian approach to multi-oriented text detection in video. Transactions on pattern analysis and machine intelligence (PAMI), 33(2):412-419.

Vijayalaksmi, S. and Punithavalli, M. (2012). A fast approach to clustering datasets using dbscan and pruning algorithms. International Journal of Computer Applications (IJCA), 60(14).

Wang, H., Rong, X., and Tian, Y. (2019). Towards accurate instance-level text spotting with guided attention. In International Conference on Multimedia and Expo (ICME), pages 994-999.

Wang, Y., Shi, C., Xiao, B., Wang, C., and Qi, C. (2018). Crf based text detection for natural scene images us- ing convolutional neural network and context information. Neurocomputing, 295:46-58.

$\mathrm{Xu}, \mathrm{H} ., \mathrm{Lu}, \mathrm{C} ., \mathrm{B}$ Berendt, R., and Jha, N. (2016). Automatic nuclei detection based on generalized laplacian of gaussian filters. Journal of biomedical and health informatics, 21(3):826-837.

Yang, H., Wang, C., Che, X., Luo, S., and Meinel, C. (2015). An improved system for real-time scene text recognition. In International Conference on Multimedia Retrieval (ACM), pages 657-660.

Zhang, C., Liang, B., Huang, Z., En, M., and Han, J. (2019). Look more than once: An accurate detector for text of arbitrary shapes. arXiv preprint:1904.06535.

Zhang, C., Yao, C., Shi, B., and Bai, X. (2015). Automatic discrimination of text and non-text natural images. In International Conference on Document Analysis and Recognition (ICDAR), pages 886-890.

Zhong, Z., Sun, L., and Huo, Q. (2018). An anchor-free region proposal network for faster r-cnn based text detection approaches. arXiv preprint:1804.09003.

Zhou, X., Yao, C., Wen, H., and Wang, Y. (2017). East: an efficient and accurate scene text detector. In International conference on Computer Vision and Pattern Recognition (CVPR), pages 5551-5560. 